\title{
Major migration Corridors of Mesoscale Ocean Eddies in the South China Sea from 1992 to 2012
}

\author{
Yunyan Du ${ }^{\mathrm{a},{ }^{*}}$, Di Wu ${ }^{\mathrm{a}}$, Fuyuan Liang ${ }^{\mathrm{b}}$, Jiawei $\mathrm{Yi}^{\mathrm{a}}$, Yang Mo ${ }^{\mathrm{a}}$, Zhigang $\mathrm{He}^{\mathrm{c}}$, Tao Pei ${ }^{\mathrm{a}}$ \\ ${ }^{a}$ State Key Laboratory of Resources and Environmental Information System, Institute \\ of Geographic Sciences and Natural Resources Research, Chinese Academy of \\ Sciences, Beijing, China \\ ${ }^{\mathrm{b}}$ Department of Geography, Western Illinois University, Macomb, USA \\ ${ }^{\mathrm{c}}$ College of Ocean and Earth Sciences, Xiamen University, Xiamen, China
}

${ }^{*}$ Corresponding author address: Yunyan Du, 11A, Datun Road, Chaoyang District, Beijing 100101, China.

E-mail: duyy@lreis.ac.cn 


\begin{abstract}
It has become routine to automatically identify mesoscale ocean eddies in the world's oceans and reconstruct their trajectories from remote sensing data. However, the major migration pathways along which eddies mainly propagate are not clear, particularly in the South China Sea (SCS). This study utilized a trajectory partition-and-group method to quantitatively measure and group trajectories of eddies in the SCS from 1992 to 2012 to extract their major migration corridors. The trajectories were first simplified into segments using the minimum description length (MDL) principle, their origin and destination (OD) points, and their trajectory partitions (TP) between two consecutive tracking times, respectively. The MDL-, OD-, and TP-based segments were then respectively grouped into clusters using the Density-based Spatial Clustering of Applications with Noise (DBSCAN) algorithm. Representative trajectories, i.e., the major migration corridors, were then extracted from different clusters. Results show that the MDL-based corridors are the most tenable in revealing the migration corridors of eddies in the SCS. The major MDL-based migration corridors of both anticyclonic and cyclonic eddy in the SCS mainly extend westward and show a meridional propagation toward the equator. The different major migration corridors in the northern, central, and southern SCS could be attributed to the various influences of seabed topography and/or background currents in these regions.
\end{abstract}

Key words: mesoscale ocean eddies; eddy migration pathways; trajectory clustering; South China Sea 


\section{Introduction}

The rapid accumulation of long time series remote sensing data with enhanced spatial and temporal resolutions has significantly improved our understanding of the development and evolution processes of mesoscale ocean eddies (hereafter referred to as eddies). Previous related studies can generally be categorized into three groups. The first group developed methods to automatically identify ocean eddies from remote sensing data (e.g. Siegel and Weiss 1997; Ari Sadarjoen and Post 2000; Chelton et al. 2011a; Doglioli et al. 2007; Faghmous et al. 2012; Isern-Fontanet et al. 2003; Morrow et al. 2004; Wang et al. 2013; Yi et al. 2014). The second group mainly focused on examining the motion and dynamic characteristics of eddies in the world's oceans (e.g. Chaigneau and Pizarro 2005; Chelton et al. 2007; Chen et al. 2011; Henson and Thomas 2008; Nan et al. 2011; Wang 2003; Wu and Chiang 2007; Xiu et al. 2010). The last group mainly studied the evolution processes of individual ocean eddies and the changes of their thermohaline structures (e.g. Chen et al. 2010; Fang and Morrow 2003; Hu et al. 2011; Wang et al. 2008a; Wu and Chiang 2007; Yuan et al. 2007).

Motion characteristics of eddies have become one of the research foci since the 1990s. With respect to the development and extinction of ocean eddies, Chelton et al. (2007, 2011a) found that there are more births in the eastern whereas more deaths in the western basins. These results were confirmed by Xu et al. (2011), who studied 
global ocean eddies from a perspective of energy variations; they found more energy dissipation in the western basins and more energy consumption in areas with strong and returning currents.

Regarding the propagation trajectories, Morrow et al. (2004) systematically investigate trajectories of eddies in the southeastern Indian Ocean, the southeastern Atlantic Ocean, and the northeastern Pacific Ocean; they found that anticyclonic eddies tend to deflect equatorward whereas cyclonic eddies poleward, respectively. Chelton (2007, 2011a) investigated the 1992-2008 trajectories of anticyclonic and cyclonic eddies in the global ocean and found that eddies mainly propagate nearly due west yet with opposing meridional deflection of anticyclonic and cyclonic eddies, which confirmed the observations of Morrow et al. (2004). Similar divergent pathways of cyclonic and anticyclonic eddies were also identified off Peru (Chaigneau et al. 2008) and in the Mediterranean Sea Barbosa (Aguiar et al. 2013).

The South China Sea (SCS) is the largest semi-enclosed marginal sea in the western tropic Pacific with a total area of 3.5 million $\mathrm{km}^{2}$ and an average depth of $2000 \mathrm{~m}$. Many studies have been conducted to study the eddies in the SCS in the past decade from different perspectives. With respect to the motion trajectories, previous studies mainly focused on statistical analysis of eddies' propagation direction and distance. It is well documented that eddies in the SCS mainly propagate toward west with small meridional deflections (e.g. Wang et al., 2003; Lin et al. 2007; Xiu et al. 2010), which vary spatially and are also related to the eddy polarity (cyclonic or 
anticyclonic). For example, Lin et al. (2007) found that eddies in the SCS seldom cross the $17^{\circ} \mathrm{N}$ parallel. They also found that in the northern SCS (north of the $17^{\circ} \mathrm{N}$ parallel), eddies developing east of $118^{\circ} \mathrm{E}$ and next to the Luzon Strait mainly move northwestward while those in the west move westward along the $200 \mathrm{~m}$ depth isoline By contrast, eddies in the southern SCS (south of the $17^{\circ} \mathrm{N}$ parallel) mainly propagate westward then shift southward along the $2000 \mathrm{~m}$ depth isoline (Lin et al. 2007).

It has now become routine to reconstruct the migration trajectories of eddies. In a large region such as the SCS, long time series motion trajectories of ocean eddies appear disorderly distributed across the whole basin though visually there may be one or more prominent directions. In fact, motion characteristics of eddies in the SCS are very complicated in terms of their movement directions as their development and evolution processes are influenced by more than one factor, such as seabed topography, wind jet, and background currents. This study examined the distribution of eddy trajectories from a perspective different from statistical analysis. We tested three different trajectory simplification methods and then utilized the trajectory partition-and-group method (Lee et al. 2007) to cluster trajectories of eddies in the SCS by considering their spatial orientation and distribution. Finally we extracted the major migration corridors from the clustered trajectories. As presented in this article, such an analysis helps us better understand how different forces drive eddies to propagate along different major corridors in the SCS. 


\section{Data and methodology}

\section{a. Data}

Twenty years of altimetric sea level anomaly (SLA) data (1992 to 2012) were used in this study. The SLA data are a merged product of TOPEX/POSEIDON, Jason-1, Envisat and European Research Satellite (ERS) altimeter observations (http://www.aviso.oceanobs.com) (Ducet et al. 2000). With combined signals from multiple satellite missions, such a product is able to better reveal mesoscale ocean activities than any single satellite mission (Le Traon and Dibarboure 1999; Chelton and Schlax 2003; Pascual et al. 2006). The SLA maps have a 7-day temporal resolution and a $1 / 3 \times 1 / 3$ degree spatial resolution. The SLA data of the shelf areas with water depth less than $100 \mathrm{~m}$ was masked out and not used in this study due to aliases from tides and internal waves over these areas (Yuan et al. 2006).

\section{b. Ocean eddy detection and tracking methods}

In the past several years, our research team has mainly devoted our efforts to improve current methods for automatically detecting and tracking ocean eddies. We have developed a hybrid detention (HD) method to improve eddy identification and also detect the changes of eddies' internal structures (Yi et al. 2014). The HD method integrated the ideas of the O-W method (Okubo 1970; Weiss 1991) and the SSH-based method (Chelton et al. 2011a) to identify eddies from the SLA maps. The 
HD method was used in this study to detect ocean eddies by sequentially defining their core areas, centers, boundaries, and then structures as illustrated below.

Core areas of ocean eddies were first identified by delineating the sea surface areas with the Okubo-Wiess parameter $(W)$ less than $-0.2 \sigma_{\mathrm{w}}\left(\sigma_{\mathrm{w}}\right.$ is the spatial standard deviation of $W$ ). The $W$ parameter (Okubo 1970; Weiss 1991) is a function of the shearing deformation rate, the straining deformation rate, and the vorticity in the flow (see Chaigneau et al., 2008). Local extreme SLA values in the core areas were identified as eddy centers. We then produced isolines at an interval of $0.5 \mathrm{~cm}$ from the SLA maps. All shortest closed isolines that fully contain the core areas of every eddy center were extracted and scrutinized to identify whether an eddy exists as a single or a composite structure. If there is only one eddy center within the shortest closed isoline, it is defined as a "footprint" border of this single eddy. It would be defined as a "composite" border if there are at least two eddy centers within the shortest closed isoline, which accordingly shows the boundary of a composite eddy structure. For a composite eddy, a footprint border is further defined for each eddy center by identifying the outmost (longest) closed isoline within which there is only one eddy center (Yi et al. 2014).

After an eddy is detected at time $t$, a hybrid automated eddy tracking method (Yi et al. 2014) was used to pair the eddy at the next time step (time $t+1$ ). The method concatenated eddies by examining the similarity between two eddies that are located within a search radius of $150 \mathrm{~km}$ on the SLA maps at two consecutive time steps $\left(t_{\mathrm{n}}\right.$ 
and $\left.t_{\mathrm{n}+1}\right)$ in terms of their kinetic and geometric properties. A given eddy at time $t_{\mathrm{n}}$ will be paired with an eddy at time $t_{n+1}$ if there is only one eddy found within the 150 $\mathrm{km}$ radius on the SLA map at time $t_{n+1}$. When two or more eddies were found, the algorithm pairs eddies at two consecutive time steps that minimize the degree of dissimilarity. The degree of dissimilarity is measured by a dimensionless distance (Chaigneau et al. 2008).

$$
\mathrm{D}_{\mathrm{e}, \mathrm{e} 2 \mathrm{e}}=\sqrt{\left(\frac{\Delta D}{D_{0}}\right)^{2}+\left(\frac{\Delta R}{R_{0}}\right)^{2}+\left(\frac{\Delta \xi}{\xi_{0}}\right)^{2}+\left(\frac{\Delta E K E}{E K E_{0}}\right)^{2}}
$$

Where $\Delta \mathrm{D}$ is the spatial distance between the eddy pair at two consecutive time steps, $\Delta \mathrm{R}, \Delta \xi$, and $\Delta \mathrm{EKE}$ are the radius, the vorticity, and the EKE differences, respectively. $\mathrm{D}_{0}, \mathrm{R}_{0}, \xi_{0}$ and $\mathrm{EKE}_{0}$ are the characteristic length scale $\left(\mathrm{D}_{0}=100 \mathrm{~km}\right)$, characteristic radius $\left(R_{0}=50 \mathrm{~km}\right)$, characteristic vorticity $\left(\xi_{0}=10^{-6} \mathrm{~s}^{-1}\right)$, and characteristic EKE $\left(\mathrm{EKE}_{0}=100 \mathrm{~cm}^{2} \mathrm{~s}^{-2}\right)$, respectively. Specific methods to calculate these attributes are available in Chaigneau et al. (2008). When searching for the paired eddy at next time step, our hybrid tracking method only consider new born eddies (Yi et al. 2014) to minimize the chance of one eddy process jumping to another one (Chelton et al. 2011a).

The SLA maps may temporarily miss an eddy due to the weekly temporal resolution, in particular if the eddy is located in the gaps between satellite ground tracks (Chaigneau et al. 2008). Therefore an eddy existing at time $t_{n}$ may spuriously and temporarily disappear at time $t_{n+1}$ but reappear at time $t_{n+2}$. For this situation, our algorithm further searched eddies at time $t_{n+2}$ within a radius of $300 \mathrm{~km}$ from a given 
eddy at time $t_{n}$. If no eddy was found, the given eddy at time $t_{n}$ is deemed terminated. Otherwise, the afore-mentioned criteria were used to pair eddies at the $t_{n}$ and $t_{n+2}$ SLA maps.

\section{c. Trajectory clustering}

The trajectory partition-and-group method (Lee et al. 2007) was used in this study to partition and group the trajectories. The method first partitions a trajectory into a set of line segments at the characteristic points where the trajectory shows rapid changes in its behavior. Each partition is represented by a line segment between two consecutive characteristic points. The line segments should have two essentially contradictory properties: preciseness and conciseness, which ensure that as few as possible line segments are generated to best mirror the original trajectory. In this study, the preciseness is measured by the sum of the difference between the original and the simplified trajectory and the conciseness is measured by the sum of the length of a simplified trajectory. We first used the minimum description length (MDL, Lee et al., 2007) method, which ensures a minimum value of the sum of the preciseness and the conciseness, to partition the trajectory into optimal segments (Fig. 1). 


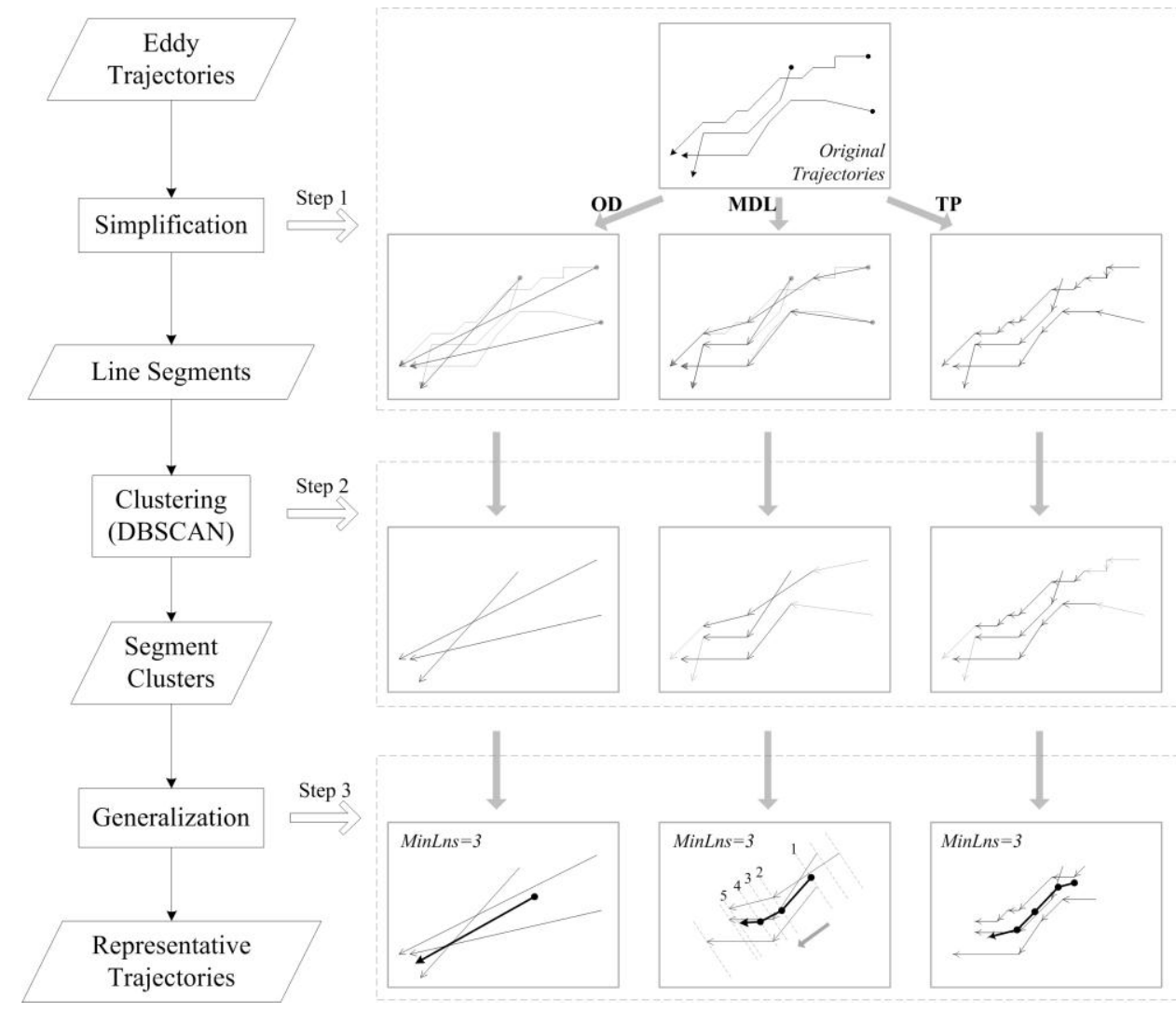

Fig. 1 A flow chart showing the process to cluster trajectories based on the minimum description length (MDL) principle, their origin and destination points (OD), and trajectory partitions (TP) between two consecutive tracking times.

Another two methods were also used in this study to simplify the trajectories: the Origin and destination points (OD) and trajectory partitions (TP) methods (Fig. 1). The former method uses a straight line segment connecting the origin and destination points of an ocean eddy to represent its trajectory whereas the latter partitions a trajectory into line segments between every two consecutive tracking times. The OD method partitions the trajectories to achieve a maximum value of the conciseness and a minimum value of the preciseness whereas the TP method achieving a maximum value of the preciseness and a minimum value of the conciseness. In other words, the 
OD and TP methods generate line segments with maximum and minimum generalization, respectively. Therefore, the OD- and TP-method-derived trajectory segments reflect the general and instantaneous migration trend during the whole life of an eddy, respectively.

The line segments were then grouped into clusters using the density-based spatial clustering of applications with noise (DBSCAN) method (Ester et al., 1996). A cluster was identified if there are at least three line segments were grouped together. Essentially, the DBSCAN method determines whether two line segments are reachable by searching the minimum number of line segments (MinLns, which is 3 in this study) within a given $\varepsilon$ distance. The distance between line segments is the sum of their perpendicular, parallel, and angular distance with different weights. For the above-mentioned three different trajectory partition methods, we used 74, 59, and 44 $\mathrm{km}$ as the threshold values of the $\varepsilon$ distance, respectively, to achieve the least amount of uncertainties of an event associated with a given probability distribution (Lee et al., 2007).

The last step is to extract the representative trajectories from all line segments that belong to the clusters (Fig. 1). The representative trajectories thus are the major migration corridors along which eddies mainly propagate in the SCS. In this study, the representative trajectory is extracted using the sweep line approach (Lee et al. 2007). Along the direction of the major axis of a cluster, a perpendicular line is used to sweep across all line segments belonging to the cluster (Fig. 1). If the sweep line hits 
no less than 3 line segments, the average coordinates of these line segments with respect to the major axis were computed and inserted as a new point of the representative trajectory. If the sweep line hits less than 3 line segments, the current point was skipped and not used to generate the representative trajectory.

Statistical analysis was performed to test whether the corridors identified were statistically significant. We used the Monte Carlo method to randomly generate line segments which are similar to the trajectory segments in terms of the total number and length. The average distance between any pair of the line segments was calculated. This experiment was repeated 1,000 times and accordingly 1,000 average distances were calculated. The Shapiro-Wilk test (Field, 2009) showed that the dataset of these 1,000 average distances is normally distributed. We then performed a t-test to check whether there is statistically significant difference between the 1,000 average distances and the average distance of every pair of trajectory partitions that were grouped into a representative trajectory (a corridor in this study).

We then calculated the supporting rate of each corridor, i.e., the ratio between the number of the trajectory segment being incorporated to generate a representative trajectory and the total number of trajectory segments in the SCS. The corridors were categorized into major and minor corridors by comparing the support rate against a threshold value of $4 \%$, the average of the maximum trajectory density of anticyclonic and cyclonic eddies per $1 / 3^{\circ} \times 1 / 3^{\circ}$ cell (Figs. 3 and 6 ). All corridors were presented in this paper, but we focused our discussion more on the major corridoes in this study. 


\section{Results}
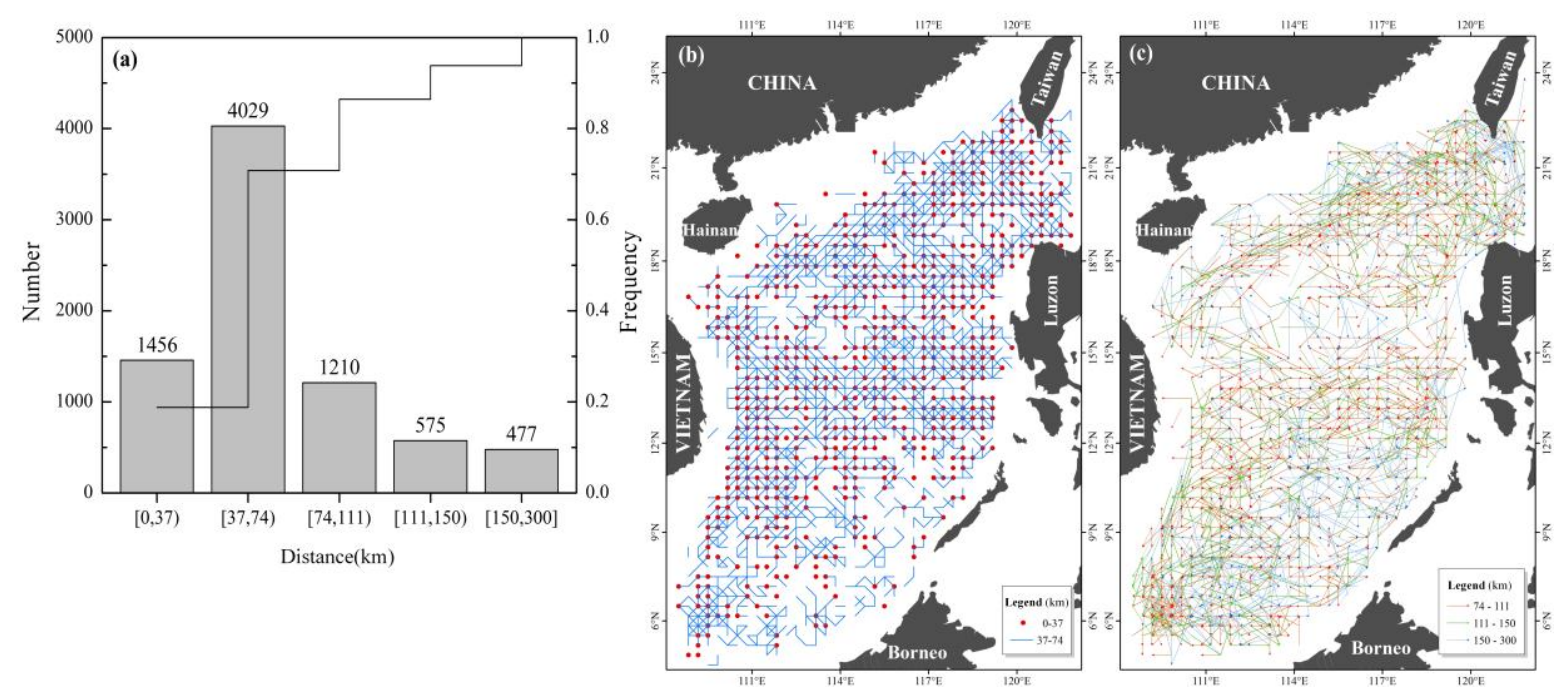

Fig. 2 (a) Histogram and cumulative distribution frequency of migration distance between two consecutive times. (b) Spatial distribution of stagnant trajectory partitions with a migration distance less than $74 \mathrm{~km}$. (c) Spatial distribution of active trajectory partitions with a migration distance more than $74 \mathrm{~km}$.

Due to the constraint of the spatial resolution of the SLA data (Chelton et al., 2011a), this study only identified the eddies with a diameter larger than $35 \mathrm{~km}$. The eddies were also observed to have a diameter at least larger than $100 \mathrm{~km}$ at one moment in their lives (Chen et al., 2011). In total, 431 anticyclonic and 449 cyclonic eddy trajectories were reconstructed from eddies which survive more than 4 weeks in the SCS from 1992 to 2012. More than 90\% eddies survive less than 100 days and migrate less than $1,000 \mathrm{~km}$ during their lifetime. In total, 7746 trajectory partitions between two consecutive observation times were identified from these 880 trajectories with 2262 longer and 5484 out of them shorter than 74 km, respectively (Fig. 2). 
Figure 2 shows the spatial distribution pattern of these trajectory segments that were investigated in this study.

\section{a. Migration corridors of anticyclonic eddies}

Figures 3-5 show the MDL-, OD-, and TP-based migration corridors of anticyclonic eddies in the SCS, respectively. The t-test results show that the existence of these corridors is all statistically significant (Table 1) at a significance level of 0.05. The MDL-based corridors are mainly located along the cells with more anticyclonic eddies in the SCS (Fig. 3).

Table 1 Results of t-test

\begin{tabular}{|c|c|c|c|}
\hline Corridors of & Simplification methods & t-value & p \\
\hline Anticyclonic eddies in Fig. 3 & MDL & 926.28 & 0.00 \\
\hline Anticyclonic eddies in Fig. 4 & OD & 917.44 & 0.00 \\
\hline Anticyclonic eddies in Fig. 5 & TP & 1047 & 0.00 \\
\hline Cyclonic eddies in Fig. 6 & MDL & 1121 & 0.00 \\
\hline Cyclonic eddies in Fig. 7 & OD & 912.24 & 0.00 \\
\hline Cyclonic eddies in Fig. 8 & TP & 1189 & 0.00 \\
\hline
\end{tabular}




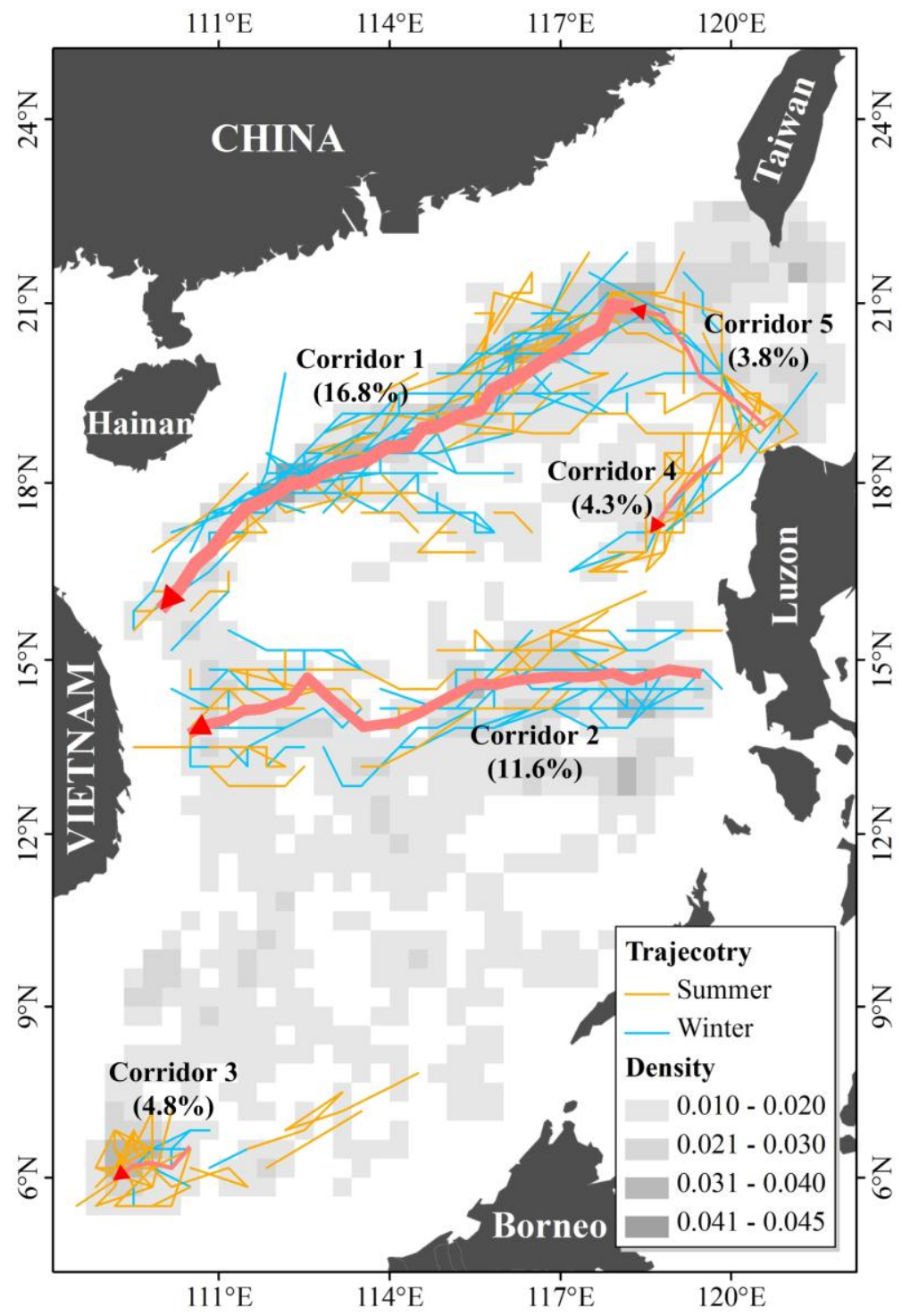

Fig. 3 Migration corridors (thick red lines) of anticyclonic eddy extracted by grouping trajectories based on MDL. The trajectory segments that contribute to the corridors are shown in blue and yellow lines nearby, which represent the segments that were found in winter and summer, respectively. The number right to a corridor shows the actual proportion of anticyclonic eddies contributing to this specific corridor. The gridded gray scale image on the background shows the density of eddy trajectory segments per cell of $1 / 3^{\circ} \times 1 / 3^{\circ}$, i.e., the ratio of the number of trajectory segments within a specific cell and the total number of trajectories in the SCS. 
Two MDL-based major (\#1 and \#4) and one minor corridors (\#5) were observed in the northern SCS (Fig. 3). The first major corridor (\#1) starts from the west of the Luzon Strait, extending toward southwest along the 2000-m-depth bathymetry isoline. Statistical analysis results show that $16.8 \%$ of the total anticyclonic eddy trajectory segments were grouped into this corridor and slightly more of them $(57.7 \%)$ were found in winter. The other two corridors are shorter and both start from the northwest of the Luzon Island, extending toward southwest (\#4) and northwest (\#5), respectively. The support rates of these two corridors are $4.3 \%$ and $3.8 \%$ respectively. The trajectory segments grouped into these two corridors were mainly found in summer (82.6\% for corridor \#4 and 70.6\% for corridor \#5). Only one major corridor (\#2 and \#3 in Fig. 3) was observed in the central and southern SCS, respectively. The corridor \#2 starts from the west of the Luzon Island $\left(15^{\circ} \mathrm{N}\right)$ and moves westward across the central SCS. The support rate of this corridor is $11.6 \%$ and slightly over half $(54.2 \%)$ of the trajectory segments grouped into this corridor are winter segments (November to next April). The corridor \#3 is much shorter (Fig. 4). It extends southwestward with a support rate of $4.8 \%$. Most of the trajectory segments $(77.3 \%)$ grouped into this corridor are summer segments (May to October). 


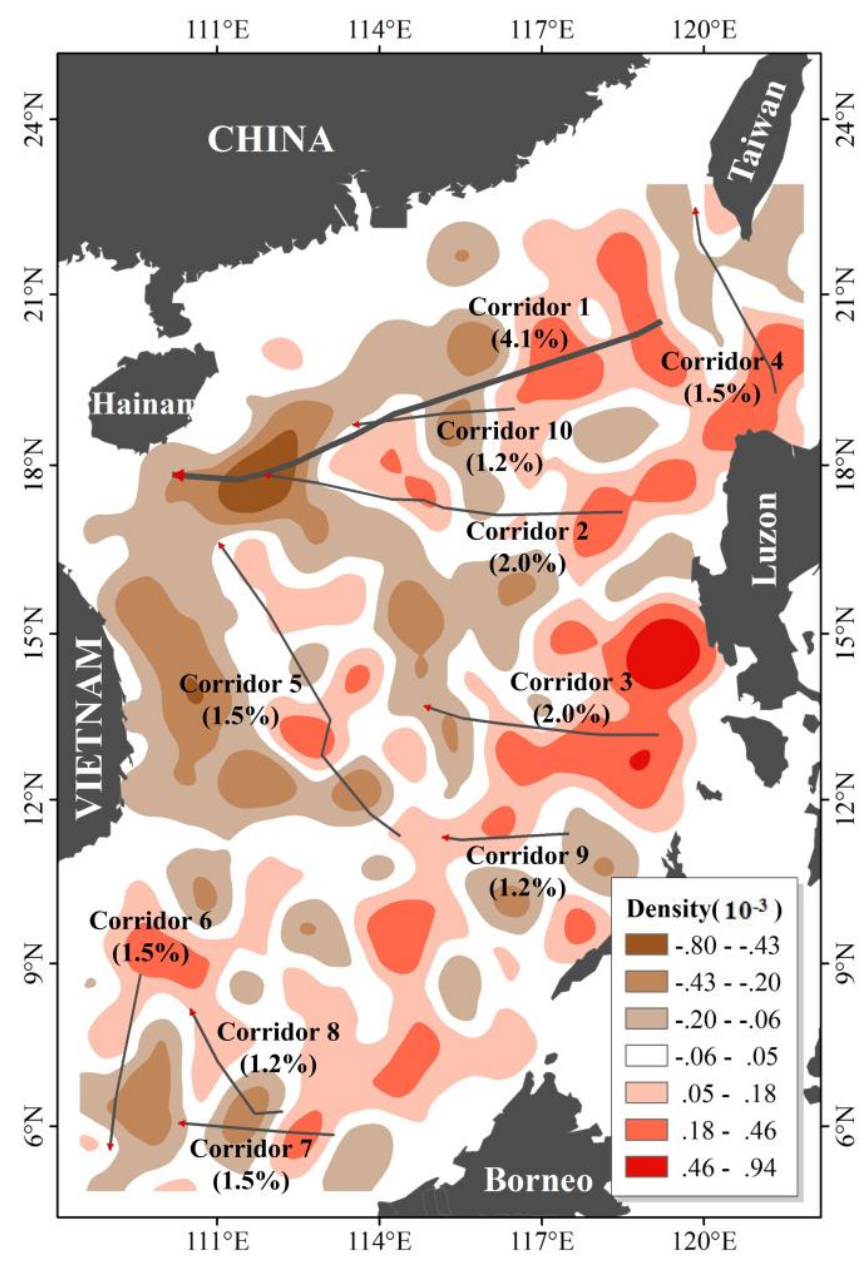

Fig. 4 Anticyclonic eddies migration corridors extracted by clustering eddy trajectories based on their OD points. The brown-red image on the background shows the difference of kernel density (a weighted magnitude-per-unit) of eddy birth and death. Higher values in red show more births of eddy than demises whereas lower values in brown show more deaths than births.

Figure 4 shows the OD-based corridor of anticyclonic eddies. The background brown-red image shows the difference of kernel density of eddy birth and death. There are more births in the eastern SCS and more deaths in the western basin. In general, the corridors mainly start from the cells with more births and end at the cells with more deaths. There is only one major corridor (\#1 in Fig. 4), which was found in 
the northern SCS with a support rate higher than $4 \%$. This major corridor starts from the Luzon Straits $\left(119^{\circ} \mathrm{E}\right.$ and $\left.21^{\circ} \mathrm{N}\right)$ and ends in the southeast of the Hainan Island $\left(111^{\circ} \mathrm{E}\right.$ and $\left.18^{\circ} \mathrm{N}\right)$. Three minor corridors (\#2, 4 and 10 in Fig. 4) were observed in the northern SCS. The Corridors 2 and 10 extend westward whereas Corridor 4 extends northward from the north of the Luzon Islands to the southwest of the Taiwan Island.

Three minor corridors were observed in the central (\#3, 5, 9 in Fig. 4) and southern SCS (\#6, 7, and 8 in Fig. 4), respectively. In the central SCS, Corridors 3 and 9 orient westward whereas Corridor 5 northwestward. The three minor corridors in the southern SCS have a support rate between $1.2 \%-1.5 \%$, extending southward, westward, and northward, respectively.

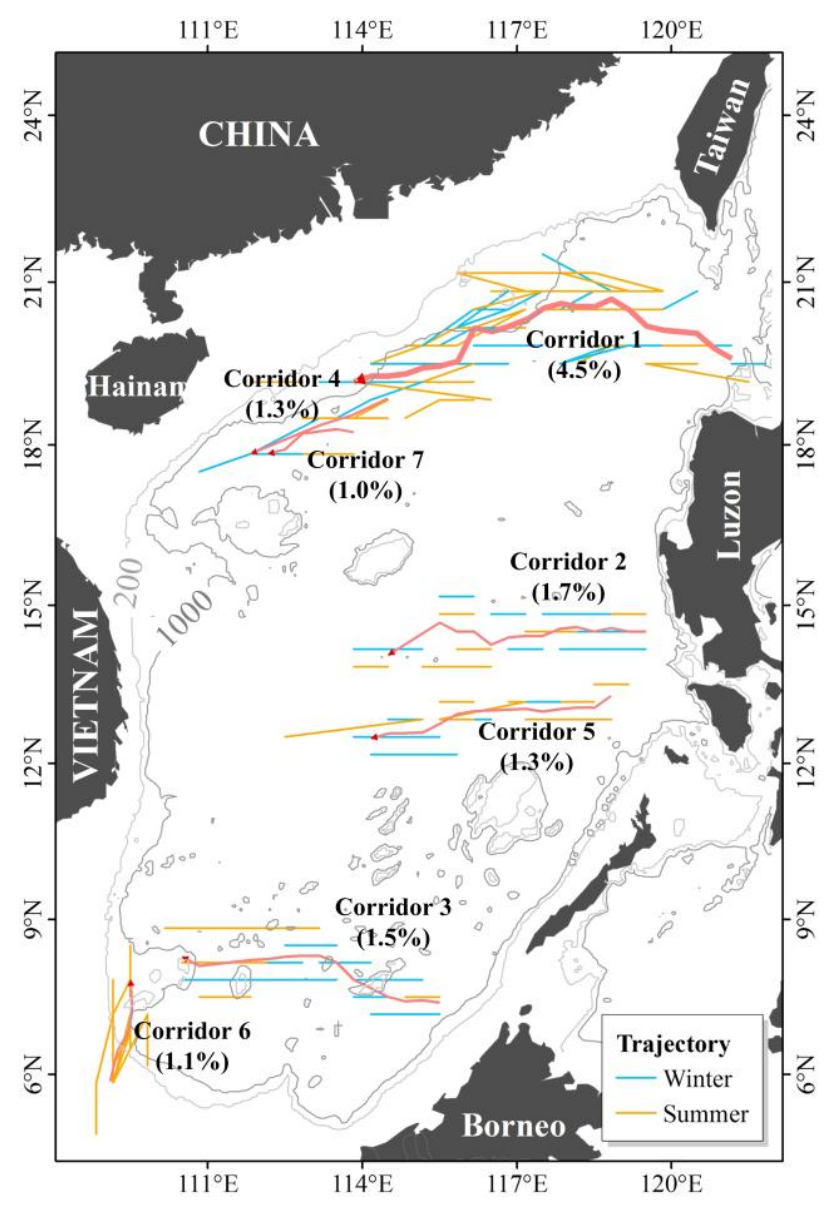


Fig. 5 Anticyclonic eddy migration corridors extracted by clustering trajectories based on their TPs. The blue and yellow lines show the winter and summer TPs that were grouped into the specified corridor, respectively.

Figure 5 shows the TP-based anticyclonic eddy migration corridors. There is only one major corridor (\#1 in Fig. 5), which was found in the northern SCS. This corridor starts from the northern mouth of the Luzon Strait and moves northwestward and then turns southwestward before it ends at $114^{\circ} \mathrm{E}$ and $19^{\circ} \mathrm{N}$. In total, $4.5 \%$ of trajectory segments of anticyclonic eddy contribute to this corridor and $64.15 \%$ of them are winter TPs. Another two minor corridors (\#4 and 7 in Fig. 5) were also found in the northern SCS. These two southwest-trending corridors are located in the north of the Dongsha Islands with a support rate of $1.0 \%$ and $1.3 \%$, respectively.

Two minor corridors (\#2, and 5 in Fig.5) were observed in the central SCS. They are generally parallel each other, both starting from west of the Luzon Island and ending at the $114^{\circ} \mathrm{E}$ meridian with a support rate of $1.3 \%$ and $1.7 \%$, respectively. In the southern SCS, one westward minor corridor (\#3 in Fig. 5) was found starting from the southeastern edge of the SCS and terminating at $8^{\circ} \mathrm{N}$ and $110.5^{\circ} \mathrm{E}$ in the Vietnam Sea. Another one (\#6 in Fig. 5) was found starting at $6^{\circ} \mathrm{N}$ and $109^{\circ} \mathrm{E}$ and extending northward along the southwestern edge of the SCS basin.

\section{b. Migration corridors of cyclonic eddies}


The MDL-, OD-, and TP-based corridors were shown in Figures 6-8. The existence of these corridors is statistically significant at a significance level of 0.05 as shown by the t-test results (Table 1). Four MDL-based corridors of cyclonic eddies were observed in the SCS and they are all located in the cells with high density of trajectory segment (Fig. 6).

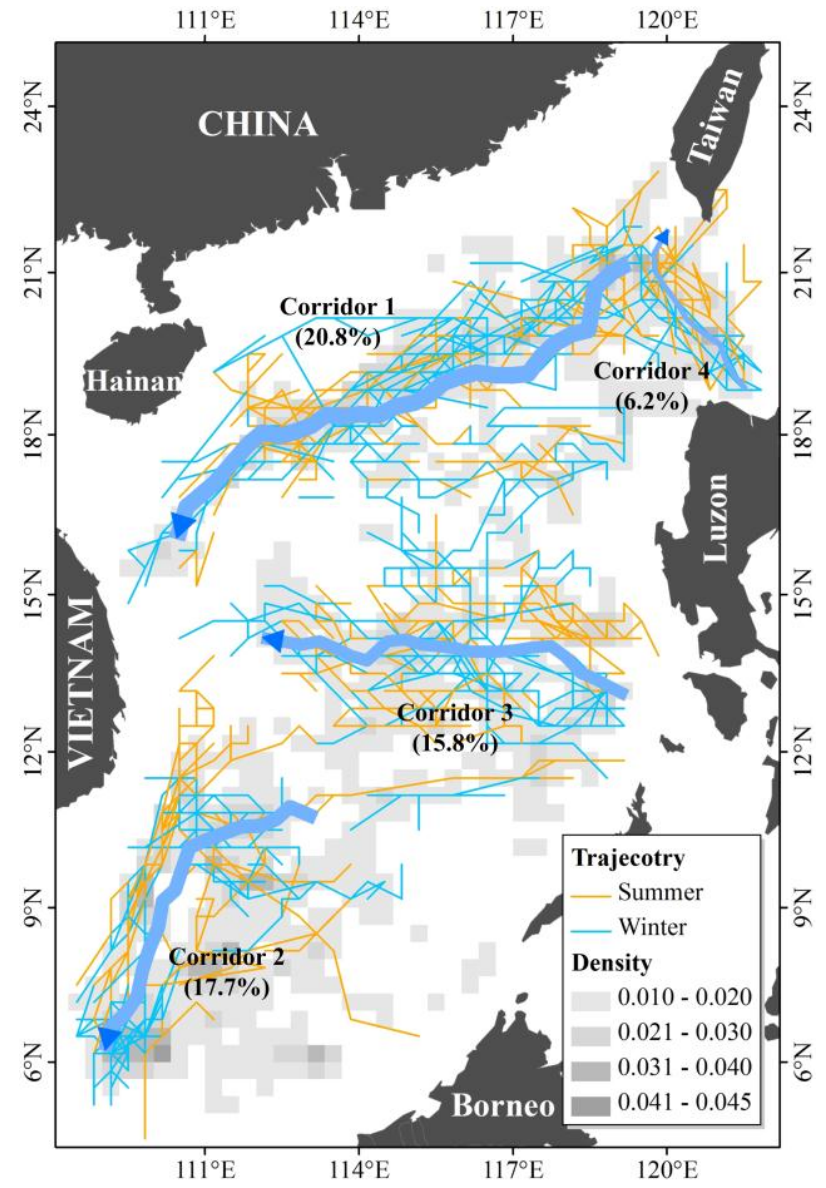

Fig. 6 Migration corridors (thick blue lines) of cyclonic eddies extracted by grouping their trajectories based on the MDL principle. Other symbols are exactly the same as those in Fig. 3. 
Two major corridors (\#1 and \#4 in Fig. 6) were observed in the northern SCS. The corridor \#1 starts from the southwest of the Taiwan Island and terminates at $16^{\circ} \mathrm{N}$ and $109^{\circ} \mathrm{E}$. In total $20.8 \%$ cyclonic eddy trajectory segments were grouped into this corridor and slightly more are winter $(60.3 \%)$ segments. The corridor \#4 has a northwest trend and a support rate of $6.2 \%$, starting from the north of the Luzon Island and ending in the southwest of the Taiwan Island. Slightly more cyclonic eddies trajectory segments grouped into this corridor are summer segments (54.6\%). In the central SCS, the major corridor \#3 was observed cutting across the SCS basin (Fig. 6). This corridor starts from the southwest of the Luzon Island and ends at $113^{\circ} \mathrm{E}$ and $14^{\circ} \mathrm{N}$. In total, $15.8 \%$ of cyclonic eddies were grouped into this corridor and more of them are summer segments $(58.3 \%)$. The corridor \#2 in the southern SCS has a southwest trend (Fig. 7), starting from $113.5^{\circ} \mathrm{E}$ and $11^{\circ} \mathrm{N}$ and ending at $109^{\circ} \mathrm{E}$ and $6^{\circ} \mathrm{N}$. Percentage of cyclonic eddies grouped into this corridor is $17.7 \%$ and slightly more of them are winter segments $(52.5 \%)$. 


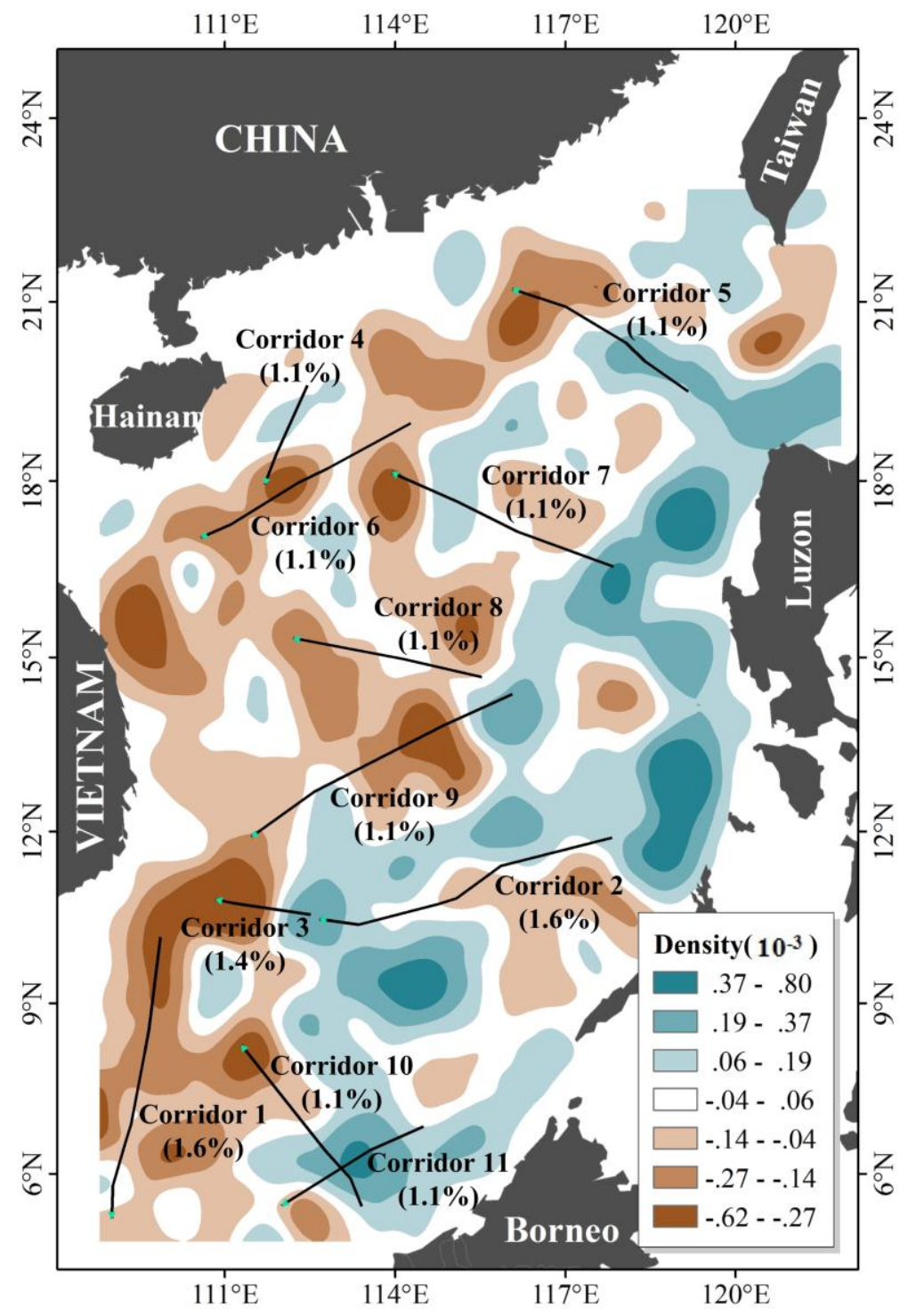

Fig. 7 Migration corridors extracted by clustering cyclonic eddy trajectories based on their OD points. The blue-brown map on the background shows the difference of kernel density of birth and death of cyclonic eddy. Higher values (dark blue) show more births than deaths while lower values (brown) show more deaths than births.

The OD-based corridors of cyclonic eddies have a support rate ranging from $1.1 \%$ to $1.6 \%$ and thus all are minor corridors (Fig. 7). In general, these minor corridors are evenly distributed across the SCS, with different trends and length. There are five 
corridors trending northwest (\#3, 5, 7, 8, and 10), five southwest (\#2, 4, 6, 9, and 11), and one south (\#1).

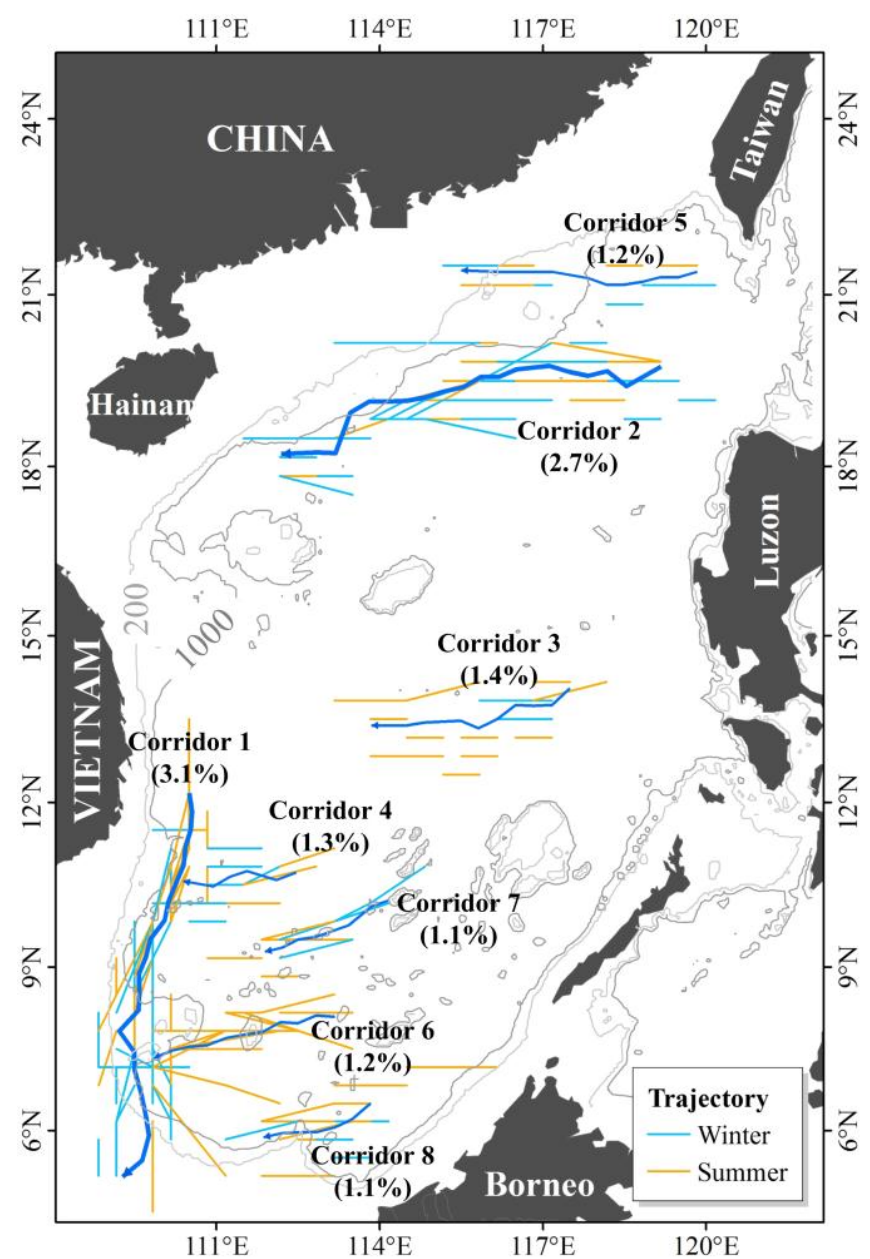

Fig. 8 Migration corridors extracted by clustering cyclonic eddy trajectory partitions.

Other symbols are exactly the same as those in Fig. 5.

All TP-based corridors of cyclonic eddies are minor corridors (Fig. 8) with a support rate ranging from $1.1 \%$ to $3.1 \%$. Two corridors (\#2 and 5 in Fig. 8) were observed in the northern SCS, extending southwestward and westward respectively. The southwestward corridor (\#2) starts from the middle outlet of the Luzon Strait and ends in the southeast of the Hainan Island. Eddies contributing to this corridor mainly 
develop in winter. The westward corridor (\#5) is generally following the $21.5^{\circ} \mathrm{N}$ parallel, starting at $120^{\circ} \mathrm{E}$ and terminating at $115^{\circ} \mathrm{E}$.

Only one short westward minor corridor (\#3 in Fig. 8) was found in the central SCS. It generally follows the $14^{\circ} \mathrm{N}$ parallel, starting at $117^{\circ} \mathrm{E}$ and ending at $114^{\circ} \mathrm{E}$. In the southern SCS, a south-trend minor corridor (\#1 in Fig. 8) was observed along the $112^{\circ} \mathrm{E}$ meridian, though the starting location slightly shifts to the east whereas the ending location to the west of the meridian. The support rate of this corridor is $3.1 \%$, the maximum value among all the corridors shown in Figure 8. There are also another four short minor corridors (\#4, 6, 7, and 8 in Fig. 8) of cyclonic eddies in the southern SCS. These four minor corridors are all westward and almost parallel each other.

\section{c. Comparison and discussion}

The most striking similarity among the MDL-, OD-, and TP-based corridors is their due-west propagation direction (Figs. 3-8), though differences are apparent. Most corridors start from the east and terminate in the west of the SCS basin. The MDL-based corridors show a dominant southwestward trend, except the two northwest-trend corridors in the northern SCS (\#5 in Fig.3 and \#4 in Fig. 6) and two westward corridors in the central SCS (\#2 in Fig. 3 and \#3 in Fig. 6). The TP-based anticyclonic and cyclonic eddy corridors were also observed in the northern, central, and southern SCS, respectively. The dominant orientation of these corridors is westward, though a north-trend anticyclonic eddy corridor (\#6 in Fig. 5) and a 
south-trend cyclonic eddy corridor (\#1 in Fig. 8) were observed in the southern SCS. By contrast, the OD- based corridors are far less organized though they also show a dominant westward orientation. Such a dominant southwestward trend suggests that both anticyclonic and cyclonic ocean eddies in the SCS mainly propagate southwestward or westward. Cai et al. (2005) also argued that most eddies in the SCS develop around major islands in the east, propagate westward, and terminate in the west.

All MDL-based corridors have a much higher support rate than the OD- and TP-based corridors. The MDL-based anticyclonic and cyclonic eddy corridors all have a support rate higher than $4 \%$ and thus are major corridors (Figs. 4 and 6), except the corridor \#5 in Fig. 3. By contrast, almost all anticyclonic and cyclonic eddy TP- and OD-based corridors have a support rate less than $4 \%$ and therefore are minor corridors (Figs 4-5 and 7-8), except the OD-based corridor \#1 in Fig. 4-5, which has a support rate $4.1 \%$. As a result, we concluded that the MDL-based corridors are more prominent and tenable in revealing the migration pattern of ocean eddies in the SCS as they are better organized and have a higher support rate. In other words, the partition-and-group method works best in identifying major corridors of ocean eddies in the SCS when their trajectories were simplified based on the MDL principle. We therefore focused on the discussion of the MDL-derived corridors hereafter unless specified otherwise. 
The corridors identified in this research confirmed the observed major migration paths in previous literatures(Fig.9). For example, Chen et al. (2011) found that eddies mainly propagate southwestward along the continental slope in the northern SCS; in a quasi-westward direction in the central SCS; with no major propagation direction in the western basin to the east of Vietnam. Compared to the verbal description of the major pathways in previous literatures, this study is the first to quantitatively measure the eddy trajectories and extract the major migration corridors of eddies in the SCS.

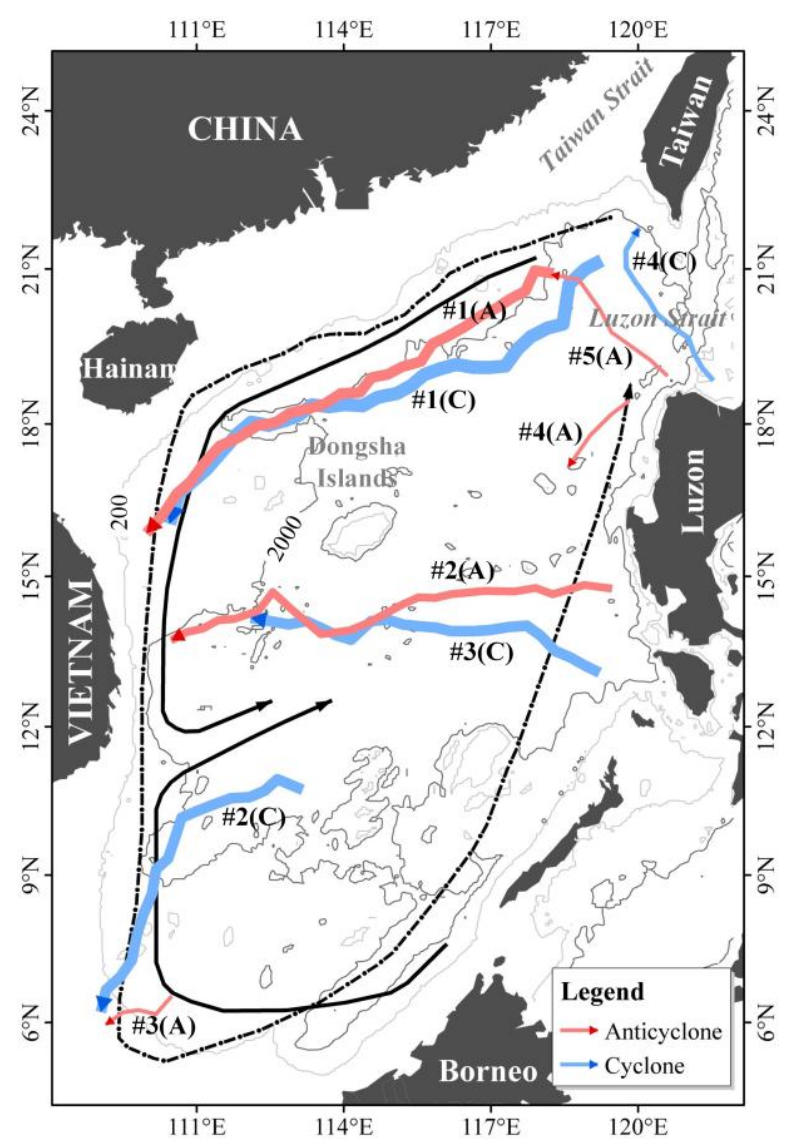

Fig. 9 Comparison of the MDL-based major corridors of anticyclonic and cyclonic eddies in the SCS. Bathymetry isolines and background currents driven by Asia monsoon were also shown to illustrate their potential influences on the migration of eddies in the SCS. 


\section{d. Influences of seabed topography and background currents}

The major migration corridors extracted in this study show that eddies in the SCS mainly propagate due west, at slightly different average speeds of $8.45,7.45$, and $10.01 \mathrm{~cm} / \mathrm{s}$ in the northern, central, and southern SCS, respectively. The corridors of both anticyclonic and cyclonic eddies meridionally extend toward equator, though their orientations vary significantly in the northern, central, and southern SCS (Fig. 9). The equator-toward corridors indicate that majority of eddies in the SCS meridionally migrate toward equator, which is inconsistent with the expected opposite meridional shift of cyclonic and anticyclonic eddies as documented in previous studies. Rossby (1948) concluded that the cyclones and anticyclones respectively show poleward and equatorward propagation due to the net meridional force resulting from the planetary gradient of the Coriolis parameter. Propagation pathways of cyclonic and anticyclonic eddies were also systematically studied, with anticyclonic eddies deflating equatorward whereas anticyclonic cyclonic eddies toward poleward in the southeast Indian, the southeast Atlantic, and the northeast Pacific Oceans (Morrow et al., 2004) and the world ocean (Chelton et al., 2011a). The inconsistency probably is due to the low latitude location of the SCS, where the $\beta$-effect is not large enough to induce the opposite meridian drift of the anticyclonic and cyclonic eddies as in mid-latitude waters (Morrow et al., 2004). Accordingly, the equator toward propagation of eddies in the SCS is more likely controlled by sea bed topography and background currents. 
The seabed topography and background currents driven by the Asia monsoon (Fig. 9) are the two factors that are frequently cited to explain the propagation pattern of the eddies in the SCS (e.g. Xiu et al., 2010; Wang et al., 2008a). The extraction of major migration corridors in this study help us better understand the influences of these two factors. In the northern SCS, the major migration corridors mainly extend southwestward, roughly along the 2000-m-depth isocline (Fig. 9), which has been reported by Lin et al. (2007), Wu and Chiang (2007), Wang et al (2008a, b), Xiu (2010) and Zhuang et al. (2010). More (63\%) trajectory segments grouped into these two corridors are winter segments and thus the orientation of these major corridors mainly mirrors that of the winter background currents (Fig. 9). The eddies in the northern SCS are most likely loaded by the background currents and mainly migrate along the major corridors \#1 (A) and \#1 (C) in Fig. 9.

In the central SCS, the major migration corridors mainly extend westward, which is totally opposite to that of the summer background currents in the SCS (Fig. 9). There are no significant winter background currents, and the seabed topography is too deep to influence the propagation of eddies in the central SCS. The westward migration of eddies in the central SCS $(7.45 \mathrm{~cm} / \mathrm{s})$ has nearly the same speed as of the long baroclinic Rossby Waves (7.1 cm/s, Yuan et al., 2007).

The migration corridors of anticyclonic and cyclonic eddies in the southern SCS mainly extend westward and southwestward, respectively. Trajectory segments grouped into these two corridors are mainly summer (77.3\% for \#3 (A) in Fig. 9) and 
winter segments (58.3\% for \#2 (C) in Fig. 9). The major migration corridors of anticyclonic and cyclonic eddies coincide with the summer and winter background currents in the southern SCS, respectively (Fig. 9), which means that they are most likely loaded by the background currents.

Understanding how these factors regulate the propagation of the eddies in the SCS is also helpful in explaining the difference among the MDL-, OD-, and TP-derived corridors, which in fact is reasonable in terms of the number and their support rates. To some extent, the TPs of an eddy reflect the "instantaneous" influences of the afore-mentioned factors and such influences may change significantly any time during the whole life of an eddy. As a result, clustering results based on the TPs tend to be less organized. The OD-derived corridors seem to oversimplify the trajectories of eddies as the movement course of an eddy was not fully considered and therefore are not able to reflect the major migration paths of ocean eddy. The MDL principle simplifies and well mirrors the original trajectories. Therefore the MDL-derived corridors reflect influences of the above-mentioned factors over a longer time period and thus are more stable and prominent.

Identification of the major eddy migration corridors is also of significance in helping us better understand relevant oceanographic processes. Many previous studies have shown that eddies can significantly impact marine biologic and biogeochemistry processes (e.g. McGillicuddy et al., 2007; Chen et al., 2008, Chenton et al., 2011b) and it is estimated that eddy-induced nutrient fluxes is responsible for $10-50 \%$ of the 
global new primary production (e.g. Siegel et al., 1999; McGillicuddy et al., 2003). In the SCS, modelling results showed that enhanced (reduced) chlorophyll, zooplankton biomass, new production, silicate update, detrital nitrogen, and silicon export are found associated with cyclonic (anticyclonic) eddies compared to the SCS basin mean. Development of an anticyclonic eddy in the ocean may deepen the thermocline and weaken the thermocline intensity whereas a cyclonic eddy may induce a shallower and thinner thermocline and strengthen the thermocline intensity (Chen et al., 2011). As a result, it is reasonable to expect the existence of major migration corridors of anticyclonic and cyclonic eddies identified in this study may show their impacts on the transport of salt and heat, as well as other marine biologic and biogeochemistry processes. However, further studies are needed to examine to what extent the existence of major migration corridors of eddies may affect the marine thermocline exchange and biogeochemistry processes in the SCS.

\section{Conclusions}

This study presented the major migration corridors of anticyclonic and cyclonic eddies in the SCS. The corridors were extracted by clustering the 1992-2012 eddy trajectories based on their MDL, OD, and TP, respectively. The MDL-derived corridors are most prominent and tenable to reveal the migration pathways of eddies 
in the SCS. Most MDL-derived corridors start from the east of the SCS basin and end up somewhere southwestward in the western SCS.

The major corridors show apparent differences between the cyclonic and anticyclonic eddies and across the SCS. In the northern SCS, both cyclonic and anticyclonic eddies show a dominant southwestward migration trend along the continental shelf. However, the cyclonic eddy corridor is located slightly northwest of the anticyclonic eddies. In the central SCS, both cyclonic and anticyclonic eddies migrate westward but the cyclonic eddy corridor starts slightly further north and head slightly southward than the anticyclonic eddies. In the southern SCS, both anticyclonic and cyclonic eddies propagate southwestward. However, the cyclonic eddy major corridor starts further north and is much longer and more prominent than that of the anticyclonic eddies.

The background currents tend to drive the eddies in the northern to propagate along the major corridors extracted in this study. The migration corridors are seemingly further steered toward south by the seabed topography. The corridors are generally parallel to the $2000 \mathrm{~m}$ depth isoline and coincide with the background currents in the northern SCS. In the southern SCS, the anticyclonic and cyclonic eddies corridors also indicate the influences of summer and winter background currents, respectively. By contrast, the eddies propagating westward in the central SCS have the average propagation speed very close to that of linear Rossby waves, 
indicating migration of eddies in the central SCS is not significantly affected by the seabed topography and background currents.

\section{Acknowledgments}

This research was supported by the National Science Foundation of China (41371378 and 41071250) and the Innovation Projects of the State Key Laboratory of Resource and Environment Information System (088RA500KA), Chinese Academy of Sciences. The altimeter products were produced by Ssalto/Duacs and distributed by Aviso, with support from Cnes (http://www.aviso.oceanobs.com/duacs/). 


\section{REFERENCES}

Aguiar, A. C. B. , Á. Peliz, and X. Carton, 2013: A census of Meddies in a long-term high-resolution simulation. Prog. Oceanogr., 116, 80-94.

Ari Sadarjoen, I., and F. H. Post, 2000: Detection, quantification, and tracking of vortices using streamline geometry. Comput. Graph., 24, 333-341.

Chaigneau, A., and O. Pizarro, 2005: Eddy characteristics in the eastern South Pacific. J. Geophys. Res. -Oceans, 110.

Chaigneau, A., A. Gizolme, and C. Grados, 2008: Mesoscale eddies off Peru in altimeter records: Identification algorithms and eddy spatio-temporal patterns. Prog. Oceanogr., 79, 106-119.

Chelton, D. B., and M. G. Schlax, 2003: The Accuracies of Smoothed Sea Surface Height Fields Constructed from Tandem Satellite Altimeter Datasets. J. Atmos. Ocean. Tech., 20, 1276-1302.

Chelton, D. B., M. G. Schlax, and R. M. Samelson, 2011a: Global observations of nonlinear mesoscale eddies. Prog. Oceanogr., 91, 167-216.

Chelton, D. B., M. G. Schlax, R. M. Samelson, and R. A. de Szoeke, 2007: Global observations of large oceanic eddies. Geophys. Res. Lett., 34.

Chelton, D. B., P. Gaube, M. G. Schlax, J. J. Early, and R. M. Samelson, 2011b: The influence of nonlinear mesoscale eddies on near-surface oceanic chlorophyll. Science, 334, 328-332.

Chen, F., W. Cai, Y. Wang, Y. Rii, R. R. Bidigare, and C. R. Benitez-Nelson, 2008: The carbon dioxide system and net community production within a cyclonic eddy in the lee of Hawaii. Deep-Sea Res. Pt. II.: Topical Studies in Oceanography, 55(10), $1412-1425$. 
Chen, G., J. Gan, Q. Xie, X. Chu, D. Wang, and Y., Hou, 2012: Eddy heat and salt transports in the South China Sea and their seasonal modulations. J. Geophys. Res., 117(C5).

Chen, G., P. Xiu, and F. Chai, 2014: Physical and biological controls on the summer chlorophyll bloom to the east of Vietnam. J. Oceangr., 70(3), 323-328.

Chen, G., Y. Hou, and X. Chu, 2011: Mesoscale eddies in the South China Sea: Mean properties, spatiotemporal variability, and impact on thermohaline structure. $J$. Geophys. Res., 116.

Chen, G., Y. Hou, X. Chu, and P. Qi, 2010: Vertical structure and evolution of the Luzon Warm Eddy. Chin. J. Oceanol. Limnol., 28, 955-961.

Doglioli, A. M., B. Blanke, S. Speich, and G. Lapeyre, 2007: Tracking coherent structures in a regional ocean model with wavelet analysis: Application to Cape Basin eddies. J. Geophys. Res., 112.

Ducet, N., P. Y. Le Traon, and G. Reverdin, 2000: Global high-resolution mapping of ocean circulation from TOPEX/Poseidon and ERS-1 and -2. J. Geophys. Res., 105, 19477-19498.

Ester, M., H.-P. Kriegel, J. Sander, and X. Xu, 1996: A density-based algorithm for discovering clusters in large spatial databases with noise. Proceedings of 2nd International Conference on Knowledge Discovery and Data Mining, 226-231.

Faghmous, J. H., Y. Chamber, S. Boriah, F. Vikebø, S. Liess, M. dos Santos Mesquita, and V. Kumar, 2012: A Novel and Scalable Spatio-Temporal Technique for Ocean Eddy Monitoring. Proceedings of the Twenty-Sixth AAAI Conference on Artificial Intelligence, 281-287. 
Fang, F., and R. Morrow, 2003: Evolution, movement and decay of warm-core Leeuwin Current eddies. Deep-Sea Res. Pt. II.: Topical Studies in Oceanography, 50, 2245-2261.

Field, A., 2009: Discovering statistics using SPSS. Sage publications, 143pp.

Henson, S. A., and A. C. Thomas, 2008: A census of oceanic anticyclonic eddies in the Gulf of Alaska. Deep-Sea Res. Pt. I: Oceanographic Research Papers, 55, 163-176.

Hu, Z. Y., A. A. Petrenko, A. M. Doglioli, and I. Dekeyser, 2011: Study of a mesoscale anticyclonic eddy in the western part of the Gulf of Lion. J. Marine Syst., 88, 3-11.

Isern-Fontanet, J., E. García-Ladona, and J. Font, 2003: Identification of Marine Eddies from Altimetric Maps. J. Atmos. Ocean. Tech., 20, 772-778.

Le Traon, P. Y., and G. Dibarboure, 1999: Mesoscale Mapping Capabilities of Multiple-Satellite Altimeter Missions. J. Atmos. Ocean. Tech., 16, 1208-1223.

Lee, J.-G., J. Han, and K.-Y. Whang, 2007: Trajectory clustering: a partition-and-group framework. Proceedings of the 2007 ACM SIGMOD international conference on Management of data, 593-604.

Lin, P., F. Wang, Y. Chen, and X. Tang, 2007: Temporal and spatial variation characteristics on eddies in the South China Sea I . Statistical analyses(in Chinese with English abstract). Acta Oceanol. Sin., 29, 14-22.

McGillicuddy, D. J., et. al., 2007: Eddy/wind interactions stimulate extraordinary mid-ocean plankton blooms. Science, 316, 1021-1026. 
McGillicuddy, D. J., L. A. Anderson, S. C. Doney, and M. E. Maltrud, 2003: Eddy-driven sources and sinks of nutrients in the upper ocean: Results from a $0.1^{\circ}$ resolution model of the North Atlantic. Global Biogeochem Cy., 17(2), 1035.

Morrow, R., F. Birol, D. Griffin, and J. Sudre, 2004: Divergent pathways of cyclonic and anticyclonic ocean eddies. Geophys Res Lett, 31(L24311), doi: 10.1029/2004GL020974.

Nan, F., Z. He, H. Zhou, and D. Wang, 2011: Three long-lived anticyclonic eddies in the northern South China Sea. J. Geophys. Res., 116.

Okubo, A., 1970: Horizontal dispersion of floatable particles in the vicinity of velocity singularities such as convergences. Deep-Sea Res. Oceanogr. Abstr., 445-454.

Pascual, A., Y. Faugère, G. Larnicol, and P.-Y. Le Traon, 2006: Improved description of the ocean mesoscale variability by combining four satellite altimeters. Geophys. Res. Lett., 33, L02611.

Rossby, C. G. 1948: On displacements and intensity changes of atmospheric vortices. J. $\operatorname{mar} . \operatorname{Res} 7,175-187$.

Siegel, D. A., and J. B. Weiss, 1997: A wavelet-packet census algorithm for calculating vortex statistics. Phys. Fluids, 9, 1988-1999.

Siegel, D. A., D. J. McGillicuddy, and E. A. Fields, 1999: Mesoscale eddies, satellite altimetry, and new production in the Sargasso Sea. J. Geophys. Res., 104(13), 13359-13379.

Wang, D., H. Xu, J. Lin, and J. Hu, 2008a: Anticyclonic eddies in the northeastern South China Sea during winter 2003/2004. J. Oceangr., 64, 925-935.

Wang, G., J. Su, and P. C. Chu, 2003: Mesoscale eddies in the South China Sea observed with altimeter data. Geophys. Res. Lett., 30, 2121. 
Wang, G., D. Chen, and J. Su, 2008b: Winter Eddy Genesis in the Eastern South China Sea due to Orographic Wind Jets. J. Phys. Oceanogr., 38, 726-732.

Wang, X., Y. Du, C. Zhou, X. Fan, and J. Yi, 2013: An improved, SSH-based method to automatically identify mesoscale eddies in the ocean. J. Trop. Oceanogr., 32, $15-23$.

Weiss, J., 1991: The dynamics of enstrophy transfer in two-dimensional hydrodynamics. Physica D, 48, 273-294.

Wu, C.-R., and T.-L. Chiang, 2007: Mesoscale eddies in the northern South China Sea. Deep-Sea Res. Pt. II., 54, 1575-1588.

Xiu, P., and F. Chai, 2011: Modeled biogeochemical responses to mesoscale eddies in the South China Sea. J. Geophys. Res., 116(C10).

Xiu, P., F. Chai, L. Shi, H. Xue, and Y. Chao, 2010: A census of eddy activities in the South China Sea during 1993-2007. J. Geophys. Res., 115.

Xu, C., X.-D. Shang, and R. Huang, 2011: Estimate of eddy energy generation/dissipation rate in the world ocean from altimetry data. Ocean Dynam., 61, 525-541.

Yi, J., Y. Du, Z. He, and C. Zhou, 2014: Enhancing the accuracy of automatic eddy detection and the capability of recognizing the multi-core structures from maps of sea level anomaly. Ocean Sci., 10, 39-48.

Yuan, D., W. Han, and D. Hu, 2006: Surface Kuroshio corridor in the Luzon Strait area based from satellite remote sensing data. J. Geophys. Res., 111, C11007.

Yuan, D., W. Han, and D. Hu, 2007: Anti-cyclonic eddies northwest of Luzon in summer-fall observed by satellite altimeters. Geophys. Res. Lett., 34. 
Zhuang, W., Y. Du, D. Wang, Q. Xie, and S. Xie, 2010a: Pathways of mesoscale variability in the South China Sea. Chin. J. Oceanol. Limnol. , 28 , 1055-1067. 